\title{
Erratum: Cluster Glass Transition of Ultrasoft-Potential Fluids at High Density [Phys. Rev. Lett. 117, 165701 (2016)]
}

\author{
Ryoji Miyazaki, Takeshi Kawasaki, and Kunimasa Miyazaki \\ (Received 9 November 2016; published 2 December 2016)
}

DOI: 10.1103/PhysRevLett.117.239902

Reference [1] is an important contribution missing from the original submission. The system in Ref. [1] is identical with that which we studied and Fig. 2 in our Letter is qualitatively the same as Fig. 2 in Ref. [1]. Our main results and conclusion, i.e., finding of the cluster glass transition, anomalous slow dynamics, and higher order dynamical singularity, need no modification. We regret the oversight and thank Prof. Schmiedeberg for bringing Ref. [1] to our attention.

[1] M. Schmiedeberg, Multiple reentrant glass transitions of soft spheres at high densities: Monotonicity of the curves of constant relaxation time in jamming phase diagrams depending on temperature over pressure and pressure, Phys. Rev. E 87, 052310 (2013). 